\title{
729.
}

\section{ON A THEOREM RELATING TO CONFORMABLE FIGURES.}

[From the Proceedings of the London Mathematical Society, vol. x. (1879), pp. 143-146. Read May 8, 1879.]

Consider two plane figures, say the figure of the points $P$ referred to axes $O x, O y$, and that of the points $P^{\prime}$ referred to axes $O x^{\prime}, O y^{\prime}$; and let $x, y$ be the coordinates of $P$, and $x^{\prime}, y^{\prime}$ those of $P^{\prime}$. If the figures correspond to each other in any manner whatever, $P$ and $P^{\prime}$ being corresponding points, then we have $x^{\prime}, y^{\prime}$ each of them a function of $x, y$; and we may consider the second figure as derived from the first by altering the distance $O P$ in the ratio $\sqrt{x^{\prime 2}+y^{12}} \div \sqrt{x^{2}+y^{2}}$, and by rotating it through the angle $\tan ^{-1} \frac{y^{\prime}}{x^{\prime}}-\tan ^{-1} \frac{y}{x}$; say by the Extension $\sqrt{x^{\prime 2}+y^{\prime 2}} \div \sqrt{x^{2}+y^{2}}$, and by the Rotation $\tan ^{-1} \frac{y^{\prime}}{x^{\prime}}-\tan ^{-1} \frac{y}{x}$; where the Extension and the Rotation are each of them a determinate function of $x, y$, the coordinates of $P$.

Passing from the point $P$ to a consecutive point $Q$, the coordinates of which are $x+d x, y+d y$ (the ratio $d y \div d x$ being arbitrary), then the coordinates of the corresponding point $Q^{\prime}$ will be $x^{\prime}+d x^{\prime}, y^{\prime}+d y^{\prime}$, where

$$
d x^{\prime}=\frac{d x^{\prime}}{d x} d x+\frac{d x^{\prime}}{d y} d y, \quad d y^{\prime}=\frac{d y^{\prime}}{d x} d x+\frac{d y^{\prime}}{d y} d y .
$$

Writing $\frac{d y^{\prime}}{d x^{\prime}}$ and $\frac{d y}{d x}$ instead of $d y^{\prime} \div d x^{\prime}$ and $d y \div d x$, the expressions

$$
\sqrt{d x^{\prime 2}+d y^{\prime 2}} \div \sqrt{d x^{2}+d y^{2}}, \text { and } \tan ^{-1} \frac{d y^{\prime}}{d x^{\prime}}-\tan ^{-1} \frac{d y}{d x},
$$

will in general have values depending upon that of the arbitrary ratio $d y: d x$. But they may be independent of this ratio; viz. this is the case when $x^{\prime}, y^{\prime}$ are functions of $x, y$ such that

$$
\frac{d x^{\prime}}{d y}=-\frac{d y^{\prime}}{d x}, \quad \frac{d y^{\prime}}{d y}=\frac{d x^{\prime}}{d x} ;
$$


and the two figures are then conformable (or conjugate) figures; that is, figures similar as regards corresponding infinitesimal elements of area. We have, in this case,

$$
\sqrt{d x^{\prime 2}+d y^{\prime 2}} \div \sqrt{d x^{2}+d y^{2}}, \text { and } \tan ^{-1} \frac{d y^{\prime}}{d x^{\prime}}-\tan ^{-1} \frac{d y}{d x},
$$

each a determinate function of $x, y$, the coordinates of $P$; and we pass from the element $P Q$ to the corresponding element $P^{\prime} Q^{\prime}$ by altering the length in the ratio $\sqrt{d x^{\prime 2}+d y^{\prime 2}} \div \sqrt{d x^{2}+d y^{2}}$, and rotating the element through the angle $\tan ^{-1} \frac{d y^{\prime}}{d x^{\prime}}-\tan ^{-1} \frac{d y}{d x} ;$ say, this ratio and this angle are the Auxesis and the Streblosis respectively, these being, as already mentioned, functions of $x, y$ only.

Considering now any two conformable figures, say the figure of the points $P$, and that of the points $P^{\prime}$; we have the theorem that we can from the first figure obtain a third conformable figure by means of an Auxesis and a Streblosis which are respectively equal to the Extension and the Rotation by which the second figure is derived from the first.

In fact, if in the three figures respectively we take $x, y, x^{\prime}, y^{\prime}$, and $x^{\prime \prime}, y^{\prime \prime}$, for the coordinates of the corresponding points $P, P^{\prime}, P^{\prime \prime}$, the first and second figures are conformable: and we have therefore

$$
\frac{d x^{\prime}}{d y}=-\frac{d y^{\prime}}{d x}, \quad \frac{d y^{\prime}}{d y}=\frac{d x^{\prime}}{d x}
$$

the third figure is to have the Auxesis $\sqrt{x^{\prime 2}+y^{\prime 2}} \div \sqrt{x^{2}+y^{2}}$, and the Streblosis

$$
\tan ^{-1} \frac{y^{\prime}}{x^{\prime}}-\tan ^{-1} \frac{y}{x}
$$

viz. writing $r$ for $\sqrt{x^{2}+y^{2}}$, we ought to have

$$
\begin{aligned}
& d x^{\prime \prime}=\frac{x x^{\prime}+y y^{\prime}}{r^{2}} d x-\frac{x y^{\prime}-x^{\prime} y}{r^{2}} d y \\
& d y^{\prime \prime}=\frac{x y^{\prime}-x^{\prime} y}{r^{2}} d x+\frac{x x^{\prime}+y y^{\prime}}{r^{2}} d y
\end{aligned}
$$

and it is therefore to be shown that there exist $x^{\prime \prime}, y^{\prime \prime}$ functions of $x, y$ satisfying these relations; for, this being so, we have

$$
\frac{d x^{\prime \prime}}{d y}=-\frac{d y^{\prime \prime}}{d x}, \quad \frac{d y^{\prime \prime}}{d y}=\frac{d x^{\prime \prime}}{d x}
$$

and the third figure is thus conformable with the first.

Writing, for shortness,

$$
A=\frac{x x^{\prime}+y y^{\prime}}{r^{2}}, \quad B=-\frac{x y^{\prime}-x^{\prime} y}{r^{2}},
$$


the equations are

$$
\begin{aligned}
& d x^{\prime \prime}=A d x-B d y \\
& d y^{\prime \prime}=B d x+A d y
\end{aligned}
$$

or the conditions for the existence of the functions $x^{\prime \prime}, y^{\prime \prime}$ are

We, in fact, have

$$
\frac{d A}{d y}+\frac{d B}{d x}=0, \quad \frac{d A}{d x}-\frac{d B}{d y}=0 .
$$

$$
\begin{aligned}
\frac{d A}{d y}+\frac{d B}{d x} & =\frac{1}{r^{2}}\left\{x\left(\frac{d x^{\prime}}{d y}+\frac{d y^{\prime}}{d x}\right)+y\left(\frac{d y^{\prime}}{d y}-\frac{d x^{\prime}}{d x}\right)+2 y^{\prime}\right\}-\frac{2}{r^{4}}\left\{\left(x x^{\prime}+y y^{\prime}\right) y+\left(x y^{\prime}-x^{\prime} y\right) x\right\} \\
& =\frac{2 y^{\prime}}{r^{2}}-\frac{2}{r^{4}}\left(x^{2}+y^{2}\right) y^{\prime}=0
\end{aligned}
$$

and similarly

$$
\begin{aligned}
\frac{d A}{d x}-\frac{d B}{d y} & =\frac{1}{r^{2}}\left\{x\left(\frac{d x^{\prime}}{d x}-\frac{d y^{\prime}}{d y}\right)+y\left(\frac{d y^{\prime}}{d x}+\frac{d x^{\prime}}{d y}\right)+2 x^{\prime}\right\}-\frac{2}{r^{4}}\left\{\left(x x^{\prime}+y y^{\prime}\right) x-\left(x y^{\prime}-x^{\prime} y\right) y\right\} \\
& =\frac{2 x^{\prime}}{r^{2}}-\frac{2}{r^{4}}\left(x^{2}+y^{2}\right) x^{\prime}=0
\end{aligned}
$$

which proves the theorem.

The theorem is closely connected with the theory of the function of an imaginary variable; for, writing the conditions for the conformable figures in the form

we have

$$
\frac{d x^{\prime}}{d x}=\frac{d y^{\prime}}{d y}=F, \quad \frac{d x^{\prime}}{d y}=-\frac{d y^{\prime}}{d x}=-G,
$$

that is,

$$
\begin{aligned}
& d x^{\prime}=F d x-G d y, \\
& d y^{\prime}=G d x-F d y
\end{aligned}
$$

$$
d x^{\prime}+i d y^{\prime}=(F+i G)(d x+i d y):
$$

whence $F+i G$ is a function of $x+i y$, and then by integration $x^{\prime}+i y^{\prime}$ is also a function of $x+i y$. In one point of view, any function such as $\phi(x, y)+i \psi(x, y)$ is a function of $x+i y$, for the quantity $x+i y$ is only known by means of its real components $x, y$; that is, knowing $x+i y$, we know $x, y$, and therefore also

$$
\phi(x, y)+i \psi(x, y)
$$

and Cauchy, adopting this definition, introduced the expression "fonction monogène" of $x+i y$, to denote that which is in the more restricted (and the ordinary) sense termed a function of $x+i y$. And MM. Briot and Bouquet, in their "Théorie des fonctions elliptiques" (Paris, 1875), although not using Cauchy's expression fonction monogène, but the simple term fonction, do this under the qualification stated p. 3:"Dans tout ce qui suit, nous ne nous occuperons que des fonctions qui admettent une dérivée." Now, a function admitting of a derivative (that is, in the ordinary 
sense, a function) of the imaginary variable $z,=x+i y$, is a function such that, for a consecutive value $z^{\prime},=x+i y+d x+i d y$, we have

$$
\frac{f\left(z^{\prime}\right)-f(z)}{z^{\prime}-z}
$$

$=\mathrm{a}$ quantity independent of the ratio of the real components $d x, d y$ of the increment $d x+i d y$ of the imaginary variable. Or, what is the same thing, writing $f(z)=x^{\prime}+i y^{\prime}$, the condition in order that $x^{\prime}+i y^{\prime}$ may be a function of $x+i y$ is

$$
d x^{\prime}+i d y^{\prime}=(F+i G)(d x+i d y)
$$

where $F$ and $G$ are functions of $x$ and $y$. It is not part of the condition that $F+i G$ shall be a function of $x+i y$, and it is only a long way further on that the authors prove that this is the case (see the definition of a "fonction holomorphe," p. 14; and the proof, p. 137). The last-mentioned equation

$$
d x^{\prime}+i d y^{\prime}=(F+i G)(d x+i d y)
$$

where $F$ and $G$ are only assumed to be functions of $x$ and $y$, has, if we represent $x+i y$ by means of the point $P$ with coordinates $(x, y)$, and in like manner $x^{\prime}+i y^{\prime}$ by means of the point $P^{\prime}$ with coordinates $\left(x^{\prime}, y^{\prime}\right)$, the geometrical interpretation that the figures of the points $P$ and $P^{\prime}$ are conformable figures, that is, figures similar as regards their infinitesimal elements. The foregoing theorem in regard to the Auxesis and the Streblosis is that we can, by means of $F$ and $G$, construct a third conformable figure,-in fact, the Auxesis and the Streblosis are $=\sqrt{F^{2}+G^{2}}$ and $\tan ^{-1} \frac{G}{F}$. respectively; and, using these as an Extension and a Rotation, we have the third conformable figure $x^{\prime \prime}+i y^{\prime \prime}=(F+i G)(x+i y)$; that is, $(F+i G)(x+i y)$, and therefore also $F+i G$, is a function of $x+i y$, - and we have thus the derivative of a function of $x+i y$ as itself a function of $x+i y$.

It is to be remarked that, although the theorem of the Auxesis and the Streblosis, considered as a property of conformable figures, is not by any means geometrically self-evident, yet the foregoing analytical proof is only a proof conducted by means of real quantities, of what (admitting the theory of imaginary quantities) is in fact self-evident; viz. the analytical conclusion really is that, $F, G$ denoting functions of $x, y$, then, if $d x^{\prime}+i d y^{\prime}=\left(F^{\prime}+i G\right)(d x+i d y)$, that is, if $\left(F^{\prime}+i G\right)(d x+i d y)$ be a complete differential, then $F+i G$ is a function of $x+i y$. 